\title{
Vorwort zur fünften Auflage
}

Für den Bereich der Unternehmungsführung ist die heutige Situation des Übergangs und der Unsicherheit mit einem hohen $\mathrm{Maß}$ an Überraschungen eher als "normal« zu werten als die hochkonjunkturellen Umweltbedingungen der vergangenen Jahre, die mit Hilfe der Inflation und des Größenwachstums manche Fehlentscheidungen praktisch konsequenzlos zugelassen hatten. In der Vergangenheit hat sich jedoch wenig Gelegenheit ergeben, Unternehmungsführung unter Verhältnissen zu lernen, wie sie gegenwärtig vorherrschen und sich für die Zukunft abzeichnen.

Die eigentliche Herausforderung für die Unternehmungen besteht in der Bewältigung des Unerwarteten und nicht in der Extrapolation von Erfolgsrezepten der Vergangenheit. Die Rechtfertigung der Unternehmung liegt zunehmend in ihrer Fähigkeit, das Unerwartete, das nicht Vorhersehbare erfolgreich und effizient im Sinne des Allgemeinwohls zu meistern. An den Grundprinzipien der Führung hat sich nichts geändert; doch die Methoden und Instrumente müssen angesichts der veränderten Umweltbedingungen modifiziert werden, und es müssen andere Prioritäten gesetzt werden. In Zeiten zunehmender Beschleunigung der Veränderung und vermehrter Risikoabwägung kommt der unternehmerischen Flexibilität, der Wahrung der Handlungsfreiheit und somit der strategischen Führung der Unternehmung wesentliche Bedeutung zu.

In der instabilen Wirtschaft unserer Zeit ist die Strategie der zunehmend häufiger in Unternehmungen unterschiedlichster Größenordnung gebrauchte Begriff, um einen praktikablen Aktionskurs für die Unternehmung festzulegen und alle Tätigkeiten danach auszurichten. Die Formulierung einer unternehmerischen Vision, die ihren Niederschlag in der Unternehmungspolitik und -kultur findet, und die Integration der Strategien mit den funktionalen Politiken, der Organisation, der operativen Planung, den Motivations- und Úberwachungssystemen kennzeichnen den Prozeß der strategischen Führung der Unternehmung; es werden, mit anderen Worten, eine unternehmerische Vision in unternehmungspolitischen Grundsätzen ausgedrückt, daraus Strategien abgeleitet, diese in spezifische Handlungsrichtlinien übersetzt, eine mit ihnen kohärente Organisationsstruktur und -kultur aufgebaut und eine Reihe von Ausführungsschritten unternommen, die die Unternehmung in die gewünschte Richtung mit der gewünschten Intensität bewegen.

Die strategische Führung der Unternehmung verlangt, ausgehend von einer stärkeren Einbeziehung von Umwelt- und Wettbewerbsüberlegungen sowie der Erforschung der Kräfte, die hinter der Umwelt- und Wettbewerbsdynamik wirken, soweit sie die Unternehmung betreffen:

(1) eine unternehmerische Vision,

(2) eine Gesamtheit von unternehmungspolitischen Grundsätzen, die ihren Niederschlag in einem Leitbild finden, 
(3) die Formulierung differenzierter Strategien für die verschiedenen strategischen Geschäftseinheiten oder Produkt/Markt-Kombinationen, die Konzentration strategischer Analysen auf kritische Bereiche, ein Denken in Alternativen, Bandbreiten und Wenn-/Dann-Konstellationen, eine ausgeprägte und klare Schwerpunktbildung und einen differenzierten Ansatz in der Ressourcenzuteilung,

(4) die direkte Umsetzung der gewählten Strategien in Aktionsprogramme mit Hilfe von Direktiven für die Funktionsbereiche und regionalen Tochtergesellschaften,

(5) die strategiegerechte Gestaltung der Organisation, die Aufteilung der Unternehmungstätigkeiten auf strategische Geschäftseinheiten sowie deren Führung und Koordination im Hinblick auf die Verwirklichung der Gesamtstrategie,

(6) ein effizientes Planungs-, Motivations- und Uberwachungssystem für die Durchführung der Strategien, und

(7) eine Unternehmungskultur, die alle Mitarbeiter bewegt, sich engagiert und motiviert für die Umsetzung der Strategien einzusetzen.

Die Konzeption der Strategien ist eine Führungsaufgabe, die niemand der Leitung der Unternehmung abnehmen kann. Wohl sind vorbereitende und beratende Tätigkeiten an Stabsstellen übertragbar, aber die Festlegung der Richtung, in die sich die Unternehmung in einer mittel- bis langfristigen Perspektive entwickeln soll, kann die Unternehmungsleitung nicht an andere Personen delegieren; die strategische Planung muß so weit wie möglich in der Linienorganisation verankert sein.

Das Studium der strategischen Prozesse bedeutet nicht, die Rolle der unternehmerischen Führungskräfte einzunehmen; es kann dazu beitragen, deren Rolle besser zu verstehen, die operativen Auswirkungen der Strategien zu erfassen und zu unterstützen und ein mit der strategischen Führung der Unternehmung konsistentes Verhalten zu entwickeln. Dadurch werden die fachlichen Leistungen in den funktionalen Bereichen aufgewertet und in ihrer Wirksamkeit verbessert. Werden Fachwissen und Können der Kader nicht nur auf die spezifischen Anforderungen ihrer funktionalen Tätigkeiten ausgerichtet, sondern darüber hinaus in eine strategische Gesamtkonzeption eingefügt, sind wesentliche Bedingungen der Selbsterfüllung und Selbstentfaltung der Mitarbeiter verwirklicht, wobei gleichzeitig das betriebliche Geschehen zu einer funktionsfähigen Einheit gebracht wird.

In der modernen Unternehmung nehmen die Kader auf eine bewußte Weise an der Vorbereitung der Entscheidungen teil, für deren Ausführung sie verantwortlich sind. Die Bewußtheit der strategischen Probleme, mit denen die Unternehmungsleitung konfrontiert wird, kann deshalb die Suche nach tragfähigen, praktikablen Lösungen bereichern. Die Rolle der Führungskräfte wird somit auch auf die autonome Beurteilung der globalen Auswirkungen ihrer Vorschläge und auf die aktive konstruktive Teilnahme an der Ausarbeitung und Durchführung der Strategien ausgedehnt. Die Durchführung der Strategien fällt somit nicht nur in den Verantwortungsbereich der Unternehmungsleitung, sondern muß als gemeinsame Verantwortung aller Führungskräfte erlebt werden.

Das Werk soll eine einheitliche, in sich geschlossene Darstellung geben und dadurch das Verständnis der strategischen Führung der Unternehmung erleichtern; darüber hinaus kann die Präsentation einer integrierenden Gesamtkonzep- 
tion für die strategische Führung der Unternehmung den Führungskräften und Studenten als praktisches Denkmodell im Gesamtbereich der Unternehmungsführung dienen.

Strategische Unternehmungsführung ist die Art zu führen, die alle Komponenten der Führung - die unternehmerische Vision, die Unternehmungspolitik, und -kultur, die Strategien, die Direktiven für die Funktionsbereiche, die Organisation, das Motivations- und Planungssystem, das Controlling, die Produkte und Dienstleistungen, integriert und harmonisiert; sie kann als Gesamtheit der Entscheidungs- und Handlungsmethodiken bezeichnet werden, die von den Führungskräften und Mitarbeitern eingesetzt werden, damit die Unternehmung in den Marktsegmenten, in denen ihre Geschäftseinheiten operieren, eine führende Wettbewerbsposition einnehmen und ihre langfristigen Gewinnaussichten unter Einhaltung bestimmter Randbedingungen verbessern kann.

Die fünfte Auflage des Lehrbuches weist gegenüber der Vorauflage einige Änderungen auf. Hinzugefügt wurde ein Abschnitt über die Grenzen der strategischen Planung. Neu ist ebenfalls der Abschnitt über das strategische Controlling. Alle anderen Abschnitte sind unter Einbeziehung neuer Untersuchungen und Arbeitsinstrumente überarbeitet worden.

Der erste Band enthält die drei Komponenten der Führung, die mit dem "Strategischen Denken“ zusammenhängen - Vision, Unternehmungspolitik und Strategie -, während der zweite Band die vier das „Strategische Handeln“ betreffenden Komponenten - Direktiven für die Funktionsbereiche (funktionale Politiken), Organisation, Aktionspläne/Fortschrittskontrolle/Strategieüberwachung sowie Unternehmungskultur und strategische Führungskompetenz behandelt.

Mein besonderer Dank gilt der Österreichischen Nationalbank, die die Arbeit durch eine großzügige finanzielle Unterstützung ermöglicht hat. Dank sagen möchte ich auch den zahlreichen Herren aus den Unternehmungen, die keine Mühe gescheut haben, meine vielen Fragen zu beantworten. Für die kritische Durchsicht des Werkes bei der Drucklegung möchte ich meinen früheren wissenschaftlichen Mitarbeitern, den Professoren Dr. R. Hammer, Dr. H. Hübner und Dr. W. Schertler, danken. Meine technische Assistentin, Frau A. Haller, meine Assistenten und Assistentinnen, die Herren Mag. M. Casagranda, Dr. G. Langer, Mag. K.-H. Lauda, Mag. H. Pechlaner, Frau Dr. M. Stumpf und Mag. J. Wörndl-Aichrieder haben mich bei der Vorbereitung der fünften Auflage der ,Strategischen Unternehmungsführung“ wesentlich unterstützt; ihnen allen gebührt herzlicher Dank. Dank gesagt sei auch dem Verlag Walter de Gruyter in Berlin für die sorgfältige Drucklegung des Werkes. 
